\title{
Ultrafast Photolysis of o-Nitrophenol Studied by Time-Resolved Photoelectron Spectroscopy
}

\author{
Yuki Nitta $^{1}$, Oliver Schalk ${ }^{2}$, Keisuke Kaneshima ${ }^{1}$, Taro Sekikawa ${ }^{1,}{ }^{*}$ \\ ${ }^{1}$ Department of Applied Physics, Hokkaido University, Kita 13 Nishi 8, Kita-ku, Sapporo 060-8628, \\ Japan \\ ${ }^{2}$ AlbaNova University Centre, Stockholm University, Roslagstullsbacken 21, 10691 Stockholm, \\ Sweden
}

\begin{abstract}
The formation of nitrous acid (HONO) through photolysis of onitrophenol was captured in real time by time-resolved photoelectron spectroscopy using single-order high harmonic probe pulses. HONO is released $250 \mathrm{fs}$ after photoexcitation.
\end{abstract}

\section{Introduction}

Volatile organic compounds (VOC) are molecules that are emitted into the atmosphere by human activities and that "cause damage to audible or visual senses" [1]. Among them, aromatic hydrocarbons form an important class and could account for more than $25 \%$ of the total VOC-amount produced in urban areas [2]. One of these molecules which received special attention is ortho-nitrophenol (o-NP). It absorbs strongly in the atmospherically important UV-A region between 320 and $400 \mathrm{~nm}$ and is known as a source of nitrous acid (HONO) which itself is a precursor for $\mathrm{OH}$-radicals. The $\mathrm{OH}$ radical is the key oxidant in the degradation of air pollutants and one of the defining molecules in atmospheric chemistry. Most importantly, it promotes the production of ozone $\left(\mathrm{O}_{3}\right)$ and particulate matter with a diameter of $\leq 2.5 \mu \mathrm{m}$ (PM2.5) [3] and can thus be regarded as a major air pollutant in inhabited areas [4]. HONO photolysis contributes up to $60 \%$ to the $\mathrm{OH}$ production [5], yet the role of $o$-NPs in the atmosphere is not understood.

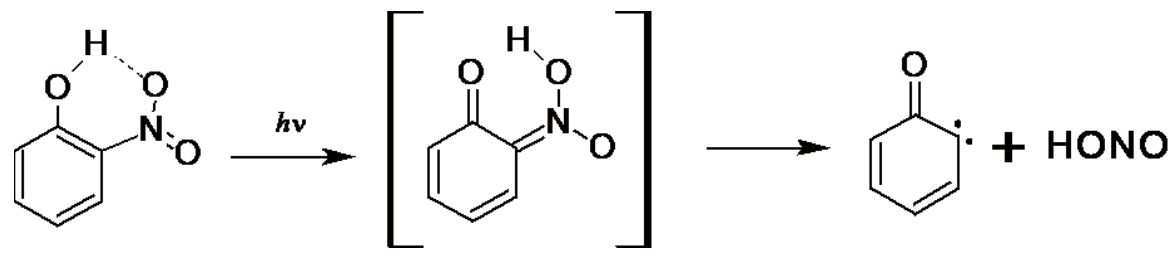

Fig. 1. Scheme of the HONO release from $o$-nitrophenol upon photoexcitation, adopted from Ref. [6].

\footnotetext{
${ }^{*}$ Corresponding author: sekikawa@eng.hokudai.ac.jp
} 
The proposed reaction scheme for the formation of HONO starting from $o$-NP is shown in Fig. 1 [6]: Upon photoexcitation, the molecule first undergoes excited state intramolecular proton transfer (ESIPT) from the hydroxyl to the nitro group. In the next step, intersystem crossing to the first excited triplet state $\left(\mathrm{T}_{2}\right)$ takes place, from where the molecule rapidly decays to the triplet ground state $\left(\mathrm{T}_{1}\right)$. In an alternative picture, the order of ESIPT and ISC is reversed [7]. In the $\mathrm{T}_{1}$ state, HONO is released. However, calculations revealed that there are several competing processes such as direct return to the ground state within the singlet manifold, and the formation of HONO has never been observed in real time [7].

In this contribution, we investigated HONO formation from $o$-NP in gas phase by timeresolved photoelectron spectroscopy, using $400 \mathrm{~nm}$ as excitation wavelength and the $19^{\text {th }}$ order of a higher-order harmonic generation (HHG) pulse $(29.5 \mathrm{eV})$ generated with a Ti:Sapphire laser pulse as a probe. For isolation of a single harmonic, we developed a timedelay compensated monochromator which also preserved the pulse duration $[8,9]$. The cross correlation of the experiment was $87 \mathrm{fs}$ as measured in krypton. For the sample, a molecular beam was prepared by first mixing $o$-NP molecules with helium gas warmed at a temperature of $323 \mathrm{~K}$. Then, the mixture was delivered to the interaction region through a heated pipe. Time-resolved photoelectron spectra were recorded with a magnetic bottle photoelectron spectrometer by changing the optical delay between the pump and probe pulses.

\section{Results and Discussion}

Figure 2a shows the photoelectron spectrum without the pump pulse (blue) and the temporal evolution of the difference spectra (pump-probe - probe only) at selected pumpprobe delays. Most prominently, we observe the rise of peaks around 11 and $12.2 \mathrm{eV}$. For a more detailed analysis, the difference spectrum taken at a delay time of $1333 \mathrm{fs}$ is replotted in Fig. 2b. We recognize the peaks at 11.0, 11.4, 12.2 and $12.6 \mathrm{eV}$. This difference spectrum agrees nicely with the photoelectron spectrum of HONO taken from Ref. [10], which is shown by the blue spectrum in Fig. $2 \mathrm{~b}$. This provides a strong indication that HONO is formed upon irradiation with 400-nm light.

The timescale of HONO formation is plotted in Fig. 2c. Here, the time evolution of the signals around 11 and $12.2 \mathrm{eV}$ is shown. The temporal behaviour of these two bands is quite similar. Directly after excitation, the intensity was reduced by the depletion of the ground state through the pump pulse and the change of the ionization cross section in the excited states. At $200 \mathrm{fs}$, the signal starts to rise and has a first maximum around $400 \mathrm{fs}$. At that delay time, the two HONO peaks are already visible (see Figure 2a). Around 600 800 fs, the spectrum becomes less intense and undergoes spectral changes, e.g. the signal around $14 \mathrm{eV}$ disappears. Afterwards, the intensity of the HONO band gradually increases again.

The temporal evolutions of the fingerprints of HONO in photoelectron spectra suggest that HONO is formed after about $250 \mathrm{fs}$. This is the first real time observation of HONO formation. The observed timescale is consistent with an earlier study by UV pump-UV probe photoelectron spectra, where the excited state dynamics is finished after about $200 \mathrm{fs}$ [11]. Since HONO is released from $T_{1}$, the disappearance of the excited states suggests the relaxation to the ground state with HONO release. In addition to HONO formation, it is evident that several other processes take place which cause a restructuring of the whole difference spectrum within the first picosecond. One of these observed processes is the rearrangement of the metastable triplet carbene formed after HONO release (see Figure 1). Moreover, the several competing processes will also be seen in the spectrum which makes the analysis quite challenging. UV pump-UV probe photoelectron spectra also revealed a 
long-lived signal that lasted for at least a ns. This agrees with our findings and indicates that most of the rearrangements seen through our difference spectra are likely ground state dynamics.
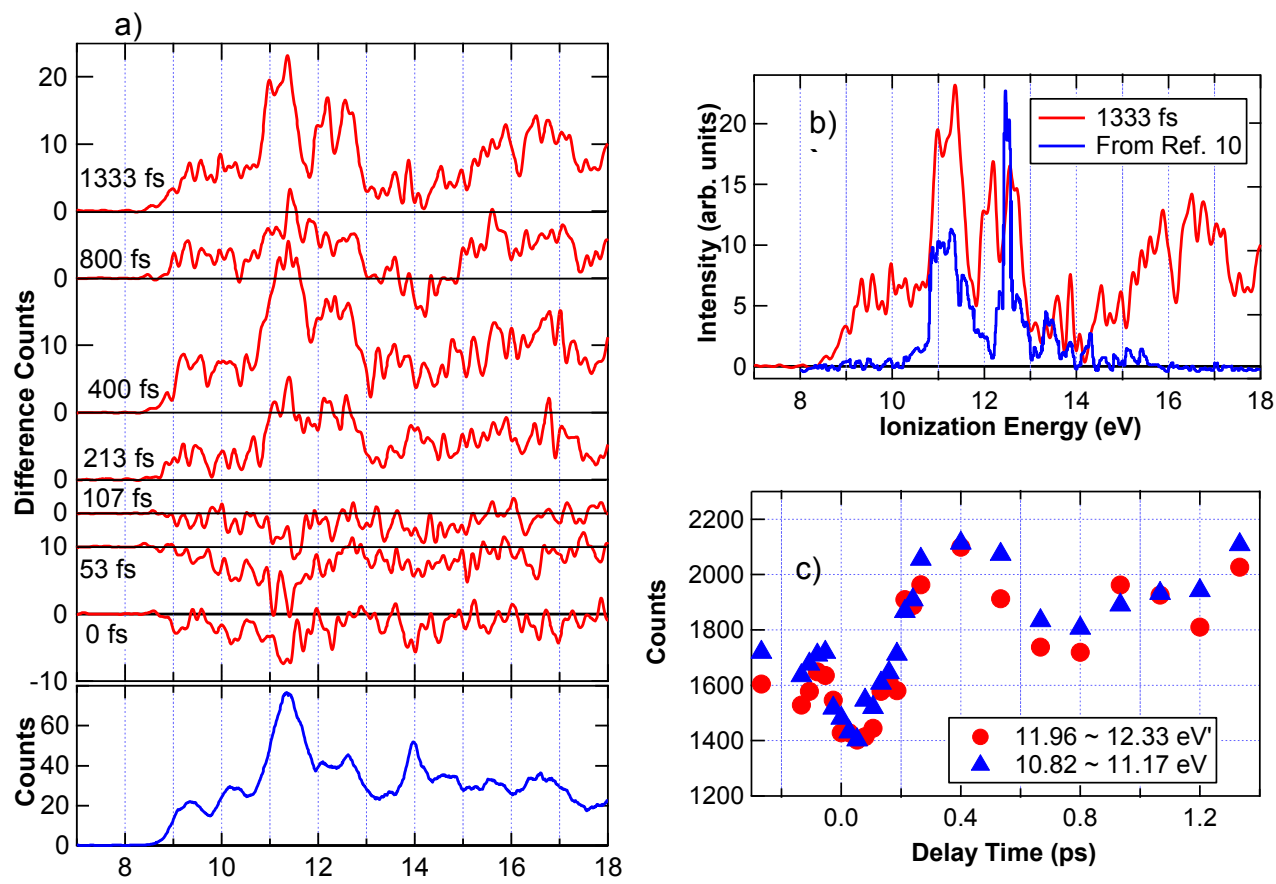

Fig. 2. a) Photoelectron spectrum of o-nitrophenol measured with a $29.5 \mathrm{eV}$ pulse only (blue curve) and temporal evolution of the difference of the photoelectron spectrum from the probe-only spectrum following excitation at $400 \mathrm{~nm}$ (red curves). b) Difference spectrum (pump-probe minus probe only) at $1333 \mathrm{fs}$ (red curve) and the photoelectron spectrum of HONO taken from Ref. [10] (blue line). c) Temporal evolution of the HONO band at $12.2(\bullet)$ and at $11 \mathrm{eV}(\boldsymbol{\Delta})$.

\section{References}

[1] Directive 2004/42/ CE of the European Parliament and of the Council of 21 April 2004.

[2] J. G. Calvert, R. Atkinson, K. H. Becker, R. M. Kamens, J. H. Seinfeld, T. J. Wallington, and G. Yarwood, The Mechanisms of Atmospheric Oxidation of the Aromatic Hydrocarbons (Oxford University Press, New York, 2002).

[3] Y. Li, J. An, M. Kajino, I. Gultepe, Y. Chen, T. Song, and J. Xin, Tellus B 67, 23930 (2015).

[4] "Health Aspects of Air Pollution with Particulate Matter, Ozone and Nitrogen Dioxide " (World Health Organization, Regional Office for Europe, Bonn, Germany 2003).

[5] B. Vogel, H. Vogel, J. Kleffmann, and R. Kurtenbach, Atmos. Environ. 37, 2957-2966 (2003).

[6] I. Bejan, Y. a. E. Aal, I. Barnes, T. Benter, B. Bohn, P. Wiesena, and J. Kleffmann, Phys. Chem. Chem. Phys. 8, 2028-2035 (2006).

[7] C. Xu, L. Yu, C. Zhu, J. Yu, and Z. Cao, Sci. Rep. 6, 26768 (2016).

[8] H. Igarashi, A. Makida, M. Ito, and T. Sekikawa, Opt. Express 20, 3725-3732 (2012).

[9] M. Ito, Y. Kataoka, T. Okamoto, M. Yamashita, and T. Sekikawa, Opt. Express 18, 6071-6078 (2010).

[10] W. Wang, M. Ge, L. Yao, X. Zeng, and Z. Wang, Chin. Sci. Bull. 52, 3056-3060 (2007).

[11] H. A. Ernst, T. J. A. Wolf, O. Schalk, N. González-García, A. E. Boguslavskiy, A. Stolow, M. Olzmann, and A.-N. Unterreiner, J. Phys. Chem. A 119, 9225-9235 (2015). 